\title{
LAGRANGIAN MODELING OF TURBULENT SPRAY COMBUSTION: APPLICATION TO ROCKET ENGINES CRYOGENIC CONDITIONS
}

\author{
J.-F. Izard and A. Mura \\ Laboratoire de Combustion et de Détonique \\ UPR 9028 du CNRS, ENSMA \\ Poitiers, BP40109, Futuroscope 86961, France
}

The present work is concerned with the application of a turbulent two-phase flow combustion model to a spray flame of Liquid Oxygen (LOx) and Gaseous Hydrogen $\left(\mathrm{GH}_{2}\right)$. The proposed strategy relies on a joint Eulerian-Lagrangian framework. The Probability Density Function $(\mathrm{PDF})$ that characterizes the liquid phase is evaluated by simulating the Williams spray equation [1] thanks to the semifluid approach introduced in [2]. The Lagrangian approach provides the classical exchange terms with the gaseous phase and, especially, several vaporization source terms. They are required to describe turbulent combustion but difficult to evaluate from the Eulerian point of view. The turbulent combustion model retained here relies on the consideration of the mixture fraction to evaluate the local fuel-to-oxidizer ratio, and the oxygen mass fraction to follow the deviations from chemical equilibrium. The difficulty associated with the estimation of a joint scalar PDF is circumvented by invoking the sudden chemistry hypothesis [3]. In this manner, the problem reduces to the estimation of the mixture fraction PDF, but with the influence of the terms related to vaporization that are the source of additional fluctuations of composition. Following the early proposal of [4], these terms are easily obtained from the Lagrangian framework adopted to describe the two-phase flows. The resulting computational model is applied to the numerical simulation of $\mathrm{LOx}-\mathrm{GH}_{2}$ spray flames. The test case (Mascotte) is representative of combustion in rocket engine conditions. The results of numerical simulations display a satisfactory agreement with available experimental data.

This is an Open Access article distributed under the terms of the Creative Commons Attribution-Noncommercial License 3.0, which permits unrestricted use, distribution, and reproduction in any noncommercial medium, provided the original work is properly cited. 


\section{INTRODUCTION}

High-performance rocket engines, such as the main engine of the European $A r$ iane launcher, usually rely on the separate injection of oxidizer and fuel, the flow velocity of the two propellant streams being fixed to reach the targeted fuel/oxidizer ratio. The dynamics of the resulting nonpremixed jet flame is controlled, to a large extent, by the levels of turbulence that are too important to be compatible with Direct Numerical Simulation (DNS) of the Navier-Stokes equations that govern the development of the reactive flowfield. Moreover, despite some recent progress towards this direction (see, for instance, [5]), the computational costs associated with the Large Eddy Simulation (LES) of such a kind of configuration are very important, and Reynolds Averaged NavierStokes (RANS) numerical simulations still remain widely used for design and optimization purposes. In fact, the two strategies are rather complimentary. Significant progress have been made in the LES direction when considering a single injector element [6], and the approach has recently allowed getting a deeper insight into such a kind of complex turbulent reactive flows. Nevertheless, the present analysis is performed within a RANS framework because of the complexity of rocket engine geometries, that contain several hundreds of injectors, still remains out of reach for LES calculations. In this respect, a great modeling effort must be done to provide reliable models capable of describing the interaction between the turbulence and chemistry in such cryogenic high-speed flows. First, it is well-known that the mean chemical production rate is significantly affected by fluctuations of temperature and concentrations so that its closure requires particular attention. Moreover, when two-phase flows are considered, the modeling difficulty is increased by the influence of the dynamics of the vaporizing spray onto the composition statistics. Experiments and theory both indicate that the presence of droplets leads to an increase in the fluctuations of temperature and composition and these fluctuations must be considered to correctly evaluate the mean chemical rate.

Despite recent developments towards the use of Eulerian descriptions for dense spray combustion (see, for instance, [7, 8]), standard Statistical Eulerian-Lagrangian (SEL) frameworks still remain the most classical way to tackle turbulent spray combustion, and such a kind of Lagrangian description is also retained for the purpose of the present study. Provided that the level of dilution is sufficiently large, the essential advantage of retaining such a Lagrangian point of view for the liquid phase is to depict most of the phenomena related to each liquid droplet (drag, vaporization, heating, etc.) by considering a single droplet in an infinite gas phase. Based on either of the two frameworks, a large amount of numerical studies devoted to the matter considered herein, i.e., combustion of $\mathrm{LOx}_{-}-\mathrm{GH}_{2}$ in rocket engine conditions have been previously conducted (see among others [7, 9-12] and, finally, [13]). These studies were essentially focused on the numerical simulation of the so-called 
A10 Mascotte test case proposed in the framework of Rocket Combustion Modelling (RCM) International Workshops. This test case is considered as one of the most pertaining and well-documented within the community. It consists of a shear coaxial cryogenic jet flame stabilized downstream of a single rocket injector element.

In previous works $[14,15]$, a nonpremixed turbulent combustion model has been implemented in the compressible and reactive Navier-Stokes solver N3SNATUR [16]. The capability of the corresponding closure of describing high-speed flow combustion has been validated against well-documented experimental data [15]. Herein, it is described how the corresponding computational model is now used in conjunction with a two-phase flow Lagrangian solver CEDILLE [17] in order to consider turbulent spray combustion. More precisely, one of the principal aims of the present study is to assess the ability of the final model to represent combustion under conditions relevant of those encountered in rocket engines. Although the operating pressures of liquid rocket engines are usually very elevated, the pressure in the combustion chamber prior to ignition remains rather low in the case of sea level ignition. In the present work, a modeling proposal capable of describing turbulent combustion of $\mathrm{LOx}-\mathrm{GH}_{2}$ propellants in such conditions is presented. The choice of available reference data to validate such a modeling proposal still remains relatively scarce and the Mascotte test rig operated at ONERA is retained as an experimental test case [18]. The numerical model relies on the consideration of the Williams equation [1] to describe the spray evolution within a Lagrangian framework together with a turbulent combustion model, which is developed and implemented within an Eulerian Navier-Stokes framework together with the classical set of Navier-Stokes equations. This results in a rather classical SEL approach of turbulent spray combustion with classical exchange terms of mass, momentum, and energy between the liquid and gaseous phases. However, the turbulent combustion model requires the determination of the mixture fraction PDF which is presumed from its first two centered moments, i. e., the mean and variance values of the mixture fraction field. Additional unknown terms associated with the vaporization process appear in the transport equations for the corresponding quantities and their closure are also to be addressed. It is noteworthy that the influence of these terms on the mixture fraction variance field is often neglected which implies that the turbulent mixing processes are very efficient with respect to those associated with the vaporization.

The present manuscript is organized as follows: first, the modeling aspects are introduced. Emphasis is placed on the description of both the two-phase flow model and the closure retained for turbulent combustion. The 10-bar Mascotte test bench and the corresponding numerical simulation results are then presented in the third and fourth sections. Finally, some conclusions and perspectives are drawn from these results in the last section. 


\section{SPRAY COMBUSTION MODEL}

\subsection{Description of Turbulent Two-Phase Flows}

In order to represent the $\mathrm{LOx}-\mathrm{GH}_{2}$ mixture, either an Eulerian or a Lagrangian framework could be retained. In the Eulerian approach, each phase is modeled as a single fluid occupying the whole physical space. In such a representation, the turbulent two-phase flows are viewed as a single mixture in which liquid and gas are considered as two species both described within an Eulerian point of view. The set of balance equations that describe the corresponding mixture can be derived by considering the so-called liquid indicator $X_{k}(\boldsymbol{x}, t)$ also often denoted phase indicator or phase function [19] defined to be 1 if $\boldsymbol{x}$ is in phase $k$ at time $t$ and 0 otherwise. Exchange terms are included to describe the transfer of mass, momentum, and energy between the two phases. Such Eulerian frameworks have been found very useful to describe primary atomization [8] or combustion in dense sprays (see, for instance, $[7,8]$ ). It must be explicitly stated that the focus of the present study will not be placed on such challenging issues associated to the description of the primary atomization itself. As a result, it is preferred to resort to a classical SEL approach. Indeed, a great deal of knowledge has been accumulated in this framework. Together with the description of the transport of the spray through convection, dispersion, etc., relevant physical models are available to describe the processes that are related to individual droplets - such as drag, vaporization, heating, etc. - as well as their interaction through secondary atomization or coalescence. The different closure strategies as well as their corresponding advantages and limitations have been already widely discussed in the literature. Further details can be found among others in the review papers [20-22].

\subsection{The Lagrangian Solver CEDILLE}

In the present approach, the Eulerian fluid solver undoubtedly remains the central part, integrating turbulence and turbulent combustion closures. Nevertheless, it seems worth introducing the general structure of the computational model, and the Lagrangian approach, as well as the physical models that are retained to represent the different phenomena involved in the spray evolution will be briefly described below. For the purpose of the present study, the key outcome of the Lagrangian solver will correspond to some vaporization terms that are detailled in the forthcoming sections.

Let us introduce the joint $\operatorname{PDF} \mathcal{F}(\boldsymbol{x}, t ; \boldsymbol{v}, r)$ with $\boldsymbol{x}$ being the position, $t$ the time, $r$ radius, and $\boldsymbol{v}$ the velocity of the particle* ${ }^{*}$ The starting point is the spray equation also often refered to as the Williams equation $[1,23]$ :

* For the sake of clarity, the temperature is first not included in the general presentation of the Lagrangian solver. 


$$
\frac{\partial \mathcal{F}}{\partial t}+\nabla_{\boldsymbol{x}}(\boldsymbol{v} \mathcal{F})+\nabla_{\boldsymbol{r}}\left(\left\langle\frac{d \boldsymbol{r}}{d t} \mid \boldsymbol{v}, r\right\rangle \mathcal{F}\right)+\nabla_{\boldsymbol{v}}\left(\left\langle\frac{d \boldsymbol{v}}{d t} \mid \boldsymbol{v}, r\right\rangle \mathcal{F}\right)=\mathcal{Q}(\mathcal{F}, \mathcal{F})
$$

In the left-hand side (LHS), the different terms correspond respectively to the temporal evolution, to the convection of a particle in the physical space, to the rate of change of characteristic size $r$, and to the acceleration. It is worth noting that a detailed consideration of the acceleration term reveals that diffusion-like terms will also arise in the right-hand side (RHS) of Eq. (1). Finally, the term $\mathcal{Q}(\mathcal{F}, \mathcal{F})$ that appears in Eq. (1) is a nonlinear quadratic term that accounts for formation and destruction of particles through the nucleation, break-up, coalescence, etc. processes. This term $\mathcal{Q}(\mathcal{F}, \mathcal{F})$ is often omitted for diluted sprays, thus leading to:

$$
\frac{\partial \mathcal{F}}{\partial t}+\nabla_{\boldsymbol{x}}(\boldsymbol{v} \mathcal{F})+\nabla_{\boldsymbol{r}}\left(\left\langle\frac{d \boldsymbol{r}}{d t} \mid \boldsymbol{v}, r\right\rangle \mathcal{F}\right)+\nabla_{\boldsymbol{v}}\left(\left\langle\frac{d \boldsymbol{v}}{d t} \mid \boldsymbol{v}, r\right\rangle \mathcal{F}\right)=0 .
$$

Since the PDF under consideration includes the velocity of the particle, the second term in the LHS does not require any further consideration since it appears in one point-one time closed form. The closure of the second term generally relies on the classical droplet vaporization model associated with the conduction limit, i.e., the well-known " $d^{2}$-law," i. e., $r^{2}(t)=r_{0}^{2}(t)-\mathcal{K}_{0} t$ where $\mathcal{K}_{0}$ is the positive constant. Nevertheless, it is also possible to resort to the generalized law introduced in [24] which aims at including the effects associated to variable properties and transient liquid heating as well as taking into account the influence of both the Stefan flow and internal recirculation. In the present work, vaporization is evaluated by using a modified " $d^{2}$-law" that includes a correction to account for convection effects, i.e., $\mathcal{K}=\mathcal{K}_{0}\left(1+0.3 \sqrt{\Re} \operatorname{Pr}^{1 / 3}\right)$ where $\Re$ and $\operatorname{Pr}$ denote the Reynolds and Prandtl numbers, respectively. Finally, the droplet acceleration is represented through a classical approach that incorporates the drag effects, i. e., the difference between the droplet velocity and the gas velocity the droplet "sees." In the corresponding model, it is assumed that the gas velocity fluctuations are distributed according to a Gaussian distribution. More details can be found in [25]. Before discussing the numerical resolution of Eq. (1), it is worth mentioning that the droplet temperature fluctuations are also taken into account, and the joint PDF $\mathcal{F}(\boldsymbol{x}, t ; \boldsymbol{v}, r, T)$ will be considered with $T$ being the droplet temperature.

From a numerical point of view, it is not possible to compute the evolution of the real number of particles involved in practical situations. Furthermore, there is no real interest in such a precise behavior of each individual particle but rather in their average behavior. In this respect, the usual trick consists in considering $n_{p}$ numerical particles that represent a group $k$ of physical particles with the same velocity, the same radius, and the same temperature at a given location in the physical space, and the joint PDF is finally obtained as 


$$
\mathcal{F}(\boldsymbol{x}, t ; \boldsymbol{v}, r, T)=\sum_{k=1}^{n_{p}} \mathcal{F}^{k}(\boldsymbol{x}, t ; \boldsymbol{v}, r, T) .
$$

Among the different methods available to perform the corresponding numerical integration, the SPM (Stochastic Particle Method) of [25] is widely used for spray calculations (see also [26]). In the present study, the semifluid proposal introduced more recently in [2] will be used. In this approach, it is considered that the velocity distribution of a class of particles at a given temperature, radius, and location is a Gaussian function. This provides a description of the turbulent two-phase flow that requires fewer degrees of freedom than classical SPM approaches, which can be of practical interest to enhance the computational efficiency. The evolution of each parcel is then evaluated thanks to the following set of Lagrangian equations:

$$
\frac{d \boldsymbol{x}_{k}}{d t}=\boldsymbol{v}_{k} ; \quad \frac{d \boldsymbol{v}_{k}}{d t}=\boldsymbol{\beta}_{k} ; \quad \frac{d T_{k}}{d t}=\mu_{k} ; \quad \frac{d r_{k}}{d t}=R_{k}
$$

where $\boldsymbol{v}_{k}, \boldsymbol{\beta}_{k}, \mu_{k}$, and $R_{k}$ incorporate the physical processes of convection, dispersion, drag, droplet heating, vaporization discussed above, and possibly atomization for situations where the spray cannot be considered as diluted any longer. The turbulent combustion model and the way it is coupled with the spray description with special emphasis on the influence of the vaporization processes will be now introduced.

\subsection{Lagrangian Model of Turbulent Combustion}

The present model of turbulent combustion is based on the original work of [27] (see also [28]). It is based on the consideration of two scalar variables: one to represent the variations of composition in the unburnt mixture (this is the mixture fraction $\xi$ defined to be zero in the pure oxidizer stream and unity in the pure fuel stream) and the other to follow the deviations from chemical equilibrium; the oxygen mass fraction, denoted $Y$, is retained to this purpose.

Scalar micromixing terms are represented with the classical IEM-LMSE model $[29,30]$ leading to the following Lagrangian evolution in the composition space $(\xi, Y)$ :

$$
\frac{d Y}{d \xi}=\frac{\widetilde{Y}-Y}{\widetilde{\xi}-\xi}+\frac{\tau \omega_{Y}}{\widetilde{\xi}-\xi}
$$

where $(\xi, Y)$ is the mean composition, and $\tau$ denotes a characteristic mixing time scale approximated by the turbulence integral time scale in the following. The second term in the RHS of Eq. (2) represents the competition between turbulent mixing and chemical reaction. 
One of the salient features of the model is that it relies on the sudden chemistry hypothesis. Indeed, within the MIL framework, it is assumed that the chemical reactions are sudden: particles need a finite time to ignite during their evolution towards the mean composition (first term in the RHS of Eq. (2)) but then instantaneously jump from the mixing line to the equilibrium line. In this manner, a strong but clearly-stated functional dependence is introduced between the two variables $\xi$ and $Y$ : the corresponding pathline $Y^{\mathrm{MIL}}(\xi)$ is fully determined through the single knowledge of the mixture fraction values that correspond to the jump positions $\xi_{\mathrm{J}-}$ and $\xi_{\mathrm{J}+}$. These values are readily obtained from a direct comparison between the mixing time scale $\tau$ and tabulated chemical time scales that can incorporate the details of the chemical kinetics [15, 31]. From the delineation of the corresponding Lagrangian path $Y=Y_{\mathrm{MIL}}(\xi)$ in the composition space, the estimation of the joint scalar $\operatorname{PDF} \widetilde{P}(Y ; \xi ; \boldsymbol{x}, t)$ can be simply expressed through the knowledge of the marginal mixture fraction $\operatorname{PDF} \widetilde{P}(\xi ; \boldsymbol{x}, t)$. This PDF shape is presumed to be a classical beta function.

\subsection{Fluctuations Induced by Vaporizing Liquid Phase}

In this manner, the present formalism simply relies on the knowledge of the mixture fraction mean $\widetilde{\xi}$ and variance $\widetilde{\xi^{\prime \prime 2}}$. Nevertheless, its extension to turbulent reactive two-phase flow situations requires additional phenomena to be considered. Indeed, if chemical reactions do not affect the mixture fraction value $\xi$, the evaporation processes that take place in such two-phase flows modify it, and additional evaporation source terms must be considered. For instance, the mixture fraction mean field follows the equation:

$$
\frac{\partial \overline{\rho \xi}}{\partial t}+\frac{\partial}{\partial x_{k}}\left(\bar{\rho} \widetilde{u}_{k} \widetilde{\xi}\right)+\frac{\partial}{\partial x_{k}}\left(\overline{\rho u_{k}^{\prime \prime} \xi^{\prime \prime}}\right)=-\overline{\rho \omega_{\text {vap }}}
$$

where the average evaporation source term $\overline{\rho \omega_{\text {vap }}}\left[\mathrm{s}^{-1}\right]$ appears with a minus sign since the liquid phase is the oxidizer for the rocket engine test cases considered below. This term exactly corresponds to the one that appears in the massconservation or continuity equation written for the gaseous phase:

$$
\frac{\partial \bar{\rho}}{\partial t}+\frac{\partial}{\partial x_{k}}\left(\bar{\rho} \widetilde{u}_{k}\right)=\overline{\rho \omega_{v a p}} .
$$

Simplest models assume that the chemical species issued from the vaporized phase is instantaneously mixed with the ambient gas. However, as proposed below, it is better to take into account the concentration inhomogeneity induced by the vaporization process: the oxygen mass fraction level is higher near the LOx droplets than far away from them. As a result, the mixture can display 
small-scale fluctuations of equivalence ratio that will influence the chemical reaction rate. These additional droplet-induced fluctuations appear naturally in the balance equation for the mixture fraction variance. Indeed, with a mixture fraction defined to be zero in the pure oxidizer and unity in the pure fuel, it writes:

$$
\begin{aligned}
\frac{\partial \overline{\rho \xi^{\prime \prime 2}}}{\partial t}+\frac{\partial}{\partial x_{k}}\left(\widetilde{u}_{k} \overline{\rho \xi^{\prime \prime 2}}\right)+\frac{\partial}{\partial x_{k}}\left(\overline{\rho u_{k}^{\prime \prime} \xi^{\prime \prime 2}}\right)=-2 \overline{\rho u_{k}^{\prime \prime} \xi^{\prime \prime}} \frac{\partial \widetilde{\xi}}{\partial x_{k}}-2 \overline{\rho D \frac{\partial \xi^{\prime \prime}}{\partial x_{k}} \frac{\partial \xi^{\prime \prime}}{\partial x_{k}}} \\
-2\left(\overline{\rho \xi \omega_{\text {vap }}}-\widetilde{\xi} \overline{\rho \omega_{\text {vap }}}\right)-\overline{\rho \xi^{2} \omega_{\text {vap }}}+\widetilde{\xi} \widetilde{\xi} \overline{\rho \omega_{\text {vap }}}
\end{aligned}
$$

In the previous transport equations, it is worth noting that molecular diffusion contributions are neglected with respect to their turbulent counterparts. The turbulence in the gas phase is evaluated from a classical $k-\epsilon$ model that provides a closure for the turbulent transport of both mean and variance of the mixture fraction field, i. e.,

$$
-\overline{\rho u_{k}^{\prime \prime} \xi^{\prime \prime}}=\bar{\rho} \frac{\nu_{T}}{\operatorname{Sc}_{\tilde{\xi}}} \frac{\partial \widetilde{\xi}}{\partial x_{k}} ; \quad-\overline{\rho u_{k}^{\prime \prime} \xi^{\prime \prime 2}}=\bar{\rho} \frac{\nu_{T}}{\operatorname{Sc}_{\widetilde{\xi^{\prime \prime 2}}}} \frac{\partial \widetilde{\xi^{\prime \prime 2}}}{\partial x_{k}}
$$

where $\nu_{T}=C_{\mu} k^{2} / \epsilon$ with $C_{\mu}=0.09$; and $\mathrm{Sc}_{\widetilde{\xi}}$ and $\mathrm{Sc}_{\widetilde{\xi^{\prime \prime 2}}}$ are the turbulent Schmidt numbers set to the usual value of 0.7 [32]. The first two terms in the RHS represent production induced by mean scalar gradients and molecular dissipation terms, respectively. The former can be expressed within the turbulent diffusivity approximation. The mean scalar dissipation rate is closed using the linear relaxation model but it must be recognized that its representation in two-phase flows still remains an open question [33]. Finally, the last four terms in the RHS are unknown correlations associated with the additional vaporization induced fluctuations.

In the present study, the liquid phase is assumed to be a spray of droplets, and the final closure is obtained by considering the Lagrangian approach introduced in [34]: the different terms are evaluated as the sum of the contributions induced by each droplet within the volume $V$ of interest:

$$
\overline{\rho \omega_{\mathrm{vap}}}=\sum_{k=1}^{n_{p}} \frac{m^{k}}{V} ; \overline{\rho \xi \omega_{\mathrm{vap}}}=\sum_{k=1}^{n_{p}} \xi_{S}^{k} \frac{m^{k}}{V} ; \overline{\rho \xi^{2} \omega_{\mathrm{vap}}}=\sum_{k=1}^{n_{p}}\left(\xi_{S}^{k}\right)^{2} \frac{m^{k}}{V}
$$

where $m^{k}$ and $\xi_{S}^{k}$ denote, respectively, the vaporized mass flow rate for the parcel $k$ and its composition taken at the liquid surface. 


\section{NUMERICAL SIMULATION}

\subsection{Experimental Test Case}

The test case retained to evaluate the performances of the model proposed for $\mathrm{LOx}-\mathrm{GH}_{2}$ nonpremixed turbulent combustion is the Mascotte facility. This experimental setup has been developed and operated over the years to facilitate the study of the fundamental processes involved in the combustion of cryogenic propellants (see [35-37] among others). An important survey of the prevailing elementary processes controlling cryogenic flames can be found in [37]; it is supported by a large amount of visualizations and quantitative measurements that have been gathered by teams of both CNRS and ONERA.

The Mascotte facility is aimed at feeding a single injector element with LOx and $\mathrm{GH}_{2}$ propellants for the test cases considered below. The first experiments were conducted from an atmospheric pressure value up to $1 \mathrm{MPa}$ with hydrogen at room temperature. A second set of experiments was performed with increased values of the hydrogen mass flow rate but keeping a subsonic flow at the injector exit; it was made possible thanks to a heat exchanger used to cool hydrogen down to $100 \mathrm{~K}$. Finally a third set of data has been also gathered for operating pressure values up to $7 \mathrm{MPa}$, i. e., above the critical pressure of oxygen. This value can be compared with the pressure value above $10 \mathrm{MPa}$ at which the Vulcain engine of the European Ariane launcher operates. It is also worth mentioning that other experiments have been conducted more recently with methane as a fuel instead of hydrogen. Here, attention is focused on the first two sets of experimental data, and the RCM-2 test case presented at the second International Workshop on Rocket Combustion Modelling (IWRCM) is considered. The test case has been designed to describe the spray diffusion flame obtained downstream of a single cryogenic coaxial injector under subcritical conditions. The cryogenic injector consists of a central pipe supplying liquid oxygen, and the overall oxidizer/fuel mass flow rate ratio is 2.11 . The injector orifice diameter for the LOx injector is $5 \mathrm{~mm}$ and it is surrounded by an annular $\mathrm{GH}_{2}$ jet with an annular channel width of $3.2 \mathrm{~mm}$. Together with the $\mathrm{OH}$ emission fields collected during the experiments, the mean and standard deviation measurements of temperature obtained with coherent anti-Stokes Raman scattering (CARS) are available at several radial and axial positions.

\subsection{Computational Model}

The Mascotte test combustor has a square internal section of $50 \times 50 \mathrm{~mm}$ but, for convenience, it was assumed that the mean flowfield is axisymmetric. The hydraulic radius of the chamber was conserved, and the numerical simulations 


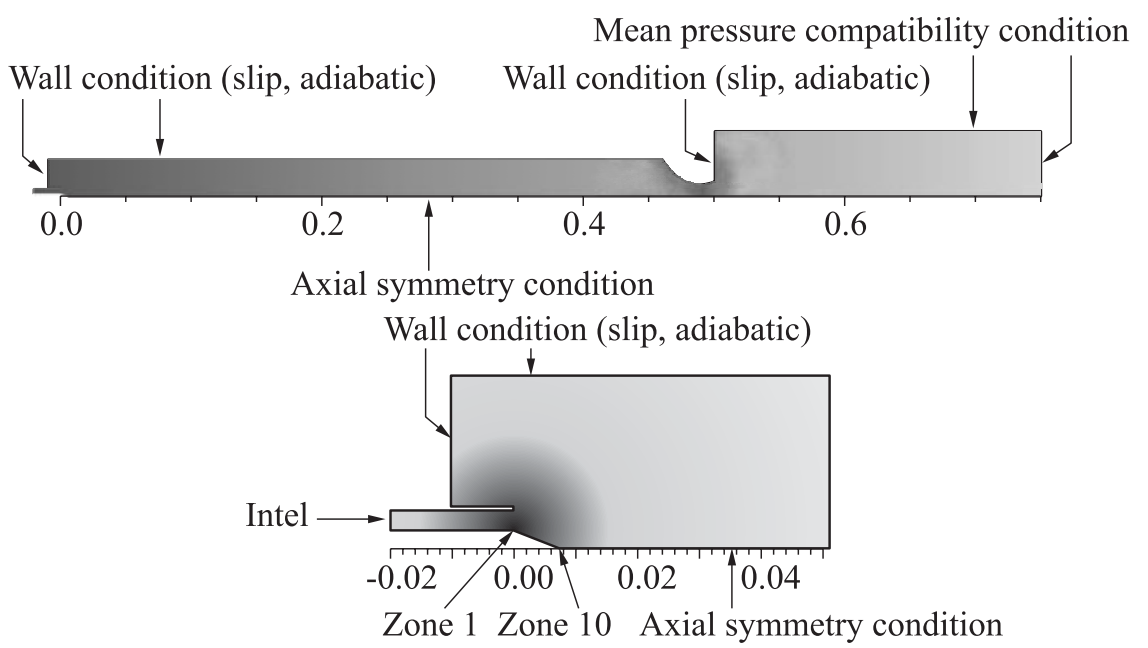

Figure 1 Computational domain and associated grid used to perform the numerical simulation of the Mascotte test case

were performed using a three-dimensional mesh corresponding to a 5 degree cut of the whole combustion chamber that was assumed to be cylindrical. The computational domain is depicted in Fig. 1, it extends over $28 \mathrm{~mm}$ in the radial direction. It was discretized using an unstructured mesh made up of 21,200 nodes and 41,150 triangular elements. It included the detailed description of the nozzle (diameter $9 \mathrm{~mm}$ and convergent length $20 \mathrm{~mm}$ ), and also featured a relatively large sponge layer downstream of the nozzle outlet. The corresponding buffer zone allowed imposing the ambient pressure level outside of the combustion chamber rather than the operating pressure of $1 \mathrm{MPa}$ itself as it was usually done (see, for instance, [8]). In this respect, it is noteworthy to mention that the reactive numerical simulations reported at the end of this section also allowed recovering an internal pressure level approximately equal to $1 \mathrm{MPa}$ but as the result of a complex balance between the injection, vaporization, combustion, and evacuation of the resulting hot gases.

Figure 1 also provides a focus on the computational mesh and geometry details in the vicinity of the injector exit. The presence of the lateral recess is expected to favor the development of a recirculation zone that will influence the flame aperture angle.

Despite some progress made in this direction (see, for instance, [7] or [8, $38]$ ), the issue associated with the development of a complete model capable of describing the two-phase flow combustion from the initial primary atomization towards the subsequent vaporization and combustion processes still remains far from being answered. In particular, the complicated problem associated with 


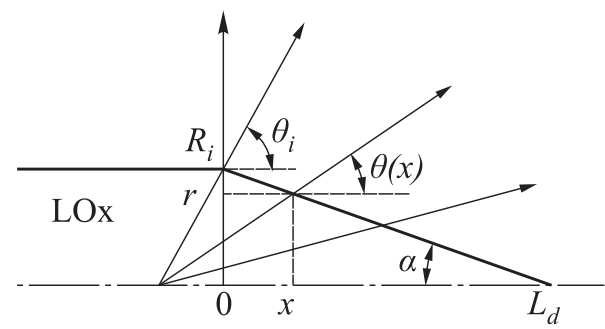

Figure 2 Geometry of the conical core and positions of injectors as modeled in [39]

the modeling of primary atomization is not addressed by the present work, and, as a result, realistic LOx droplet sizes and velocities must be prescribed at the inlet. For the present numerical simulations, the inlet conditions for droplet injection were imposed following the guidelines of the second IWRCM. They are as follows.

The LOx droplets were injected along a presumed conical core (Fig. 2), with a Sauter mean diameter value $d_{32}=82 \mu \mathrm{m}$ in agreement with the experimental data reported in [40]. This peculiar value corresponded to the one obtained after truncating at $250 \mu \mathrm{m}$ the experimental distribution measured by Phase Doppler Particle Analyzer (PDPA) thus removing a small number of very big droplets. The same value has been retained in the previous numerical studies conducted in $[9,10]$. Following the measurements performed with two-component Laser Doppler Anemometry [41], the modulus of the initial velocities of all droplets were set to $10 \mathrm{~m} / \mathrm{s}$, and the velocity components were fixed following the early proposal of $[39]$ (see also $[9,10])$.

\section{RESULTS AND DISCUSSION}

Before introducing the droplets of LOx into the computational domain, a numerical simulation of gaseous flow has been first conducted in order to converge the velocity field solution. This preliminary step of simulation was found to favor the subsequent description of the droplet transport in the physical space, providing an increased numerical convergence and stability.

Nonreactive numerical simulations of the two-phase flows were then performed from this solution, and it was found that the presence of the vaporizing spray of LOx droplets induced a significant deviation of the hydrogen stream with respect to the previous results obtained from the gaseous simulations (Fig. 3a). The mean fields of temperature and oxygen mass fraction resulting from the LOx droplet evaporation are reported in Figs. $3 c$ and $3 b$, respectively. The oxygen mass fraction (in the gas phase) reaches its maximum 


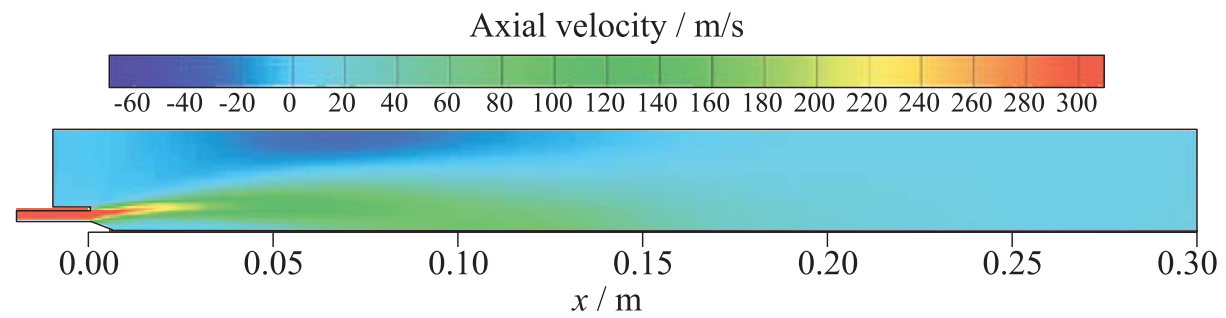

(a)

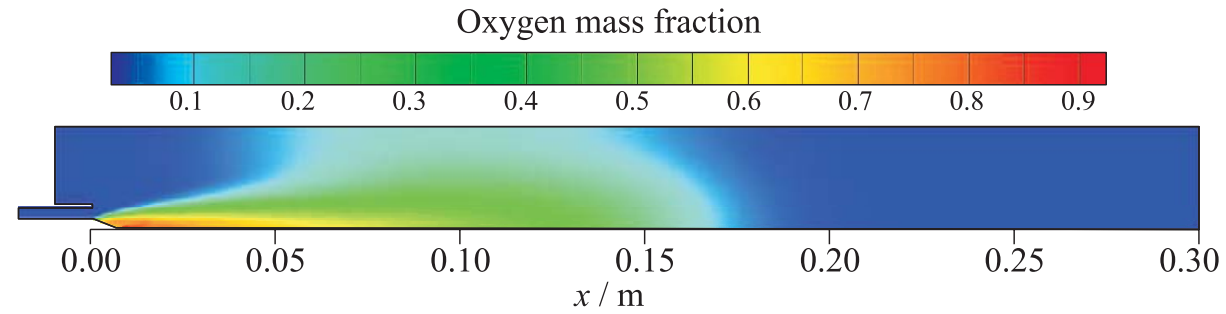

(b)

Temperature / K

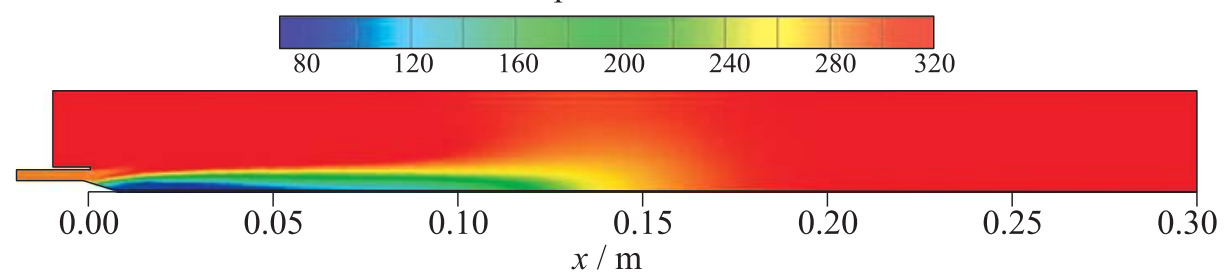

(c)

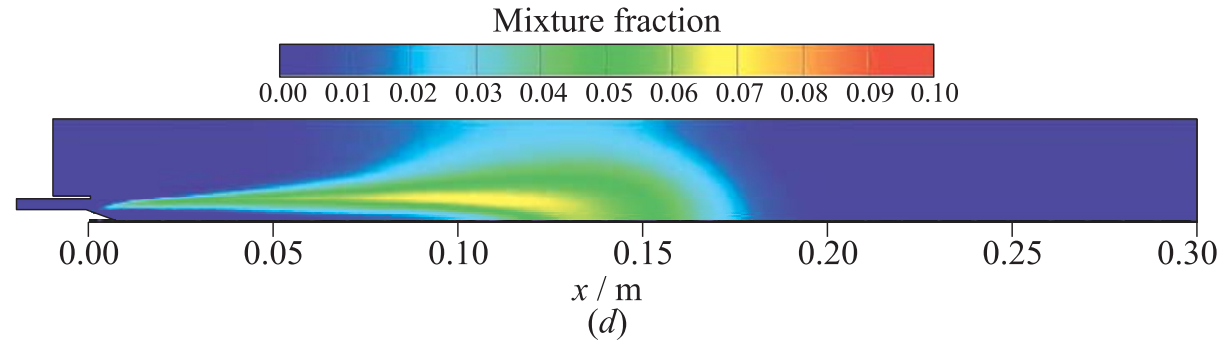

Figure 3 Mean fields of axial velocity with droplets $(a)$; oxygen mass fraction $(b)$; temperature $(c)$; and variance of the mixture fraction $(d)$ as obtained from a nonreactive simulation. (Refer Izard and Mura, p. 218.)

value in the vicinity of the symmetry axis close to the modeled liquid core. Downstream of the central LOx injector exit, the levels of axial velocity are much lower, the injected LOx droplets evaporate, and gaseous oxygen is accumulated in this zone. The temperature values remain very low, around $100 \mathrm{~K}$, 
and the mixture fraction variance levels increase only outside of this cold region (see Fig. $3 c$ ).

On the contrary, the LOx droplets that reach the shear layer between LOx and $\mathrm{GH}_{2}$ propellant streams are quickly transported far away. Keeping on evaporating, they are moved towards the combustion chamber walls in such a manner that oxygen mass fraction values are also not fully negligible in this zone. In this respect, it should be kept in mind that, because oxygen is much heavier than hydrogen, a slight variation of its local concentration induced by the vaporization process can lead to a significant increase of the corresponding mass fraction.

Finally, reactive numerical simulations of the Mascotte test case were initiated from the preliminary nonreactive step presented above. The obtained results can be first discussed in terms of both position and shape of the resulting flame. From a qualitative point of view, the experiments conducted at ONERA have always shown that the flame is attached close to the injector exit and surrounds

Mixture fraction
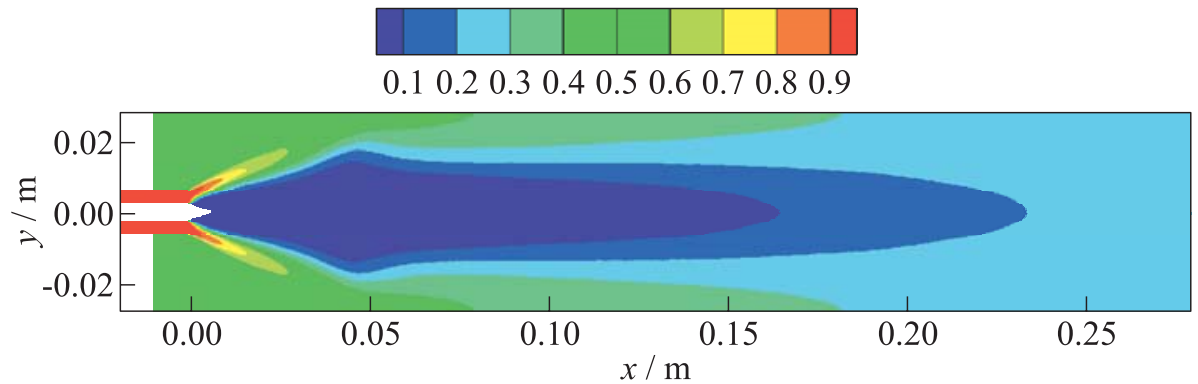

(a)

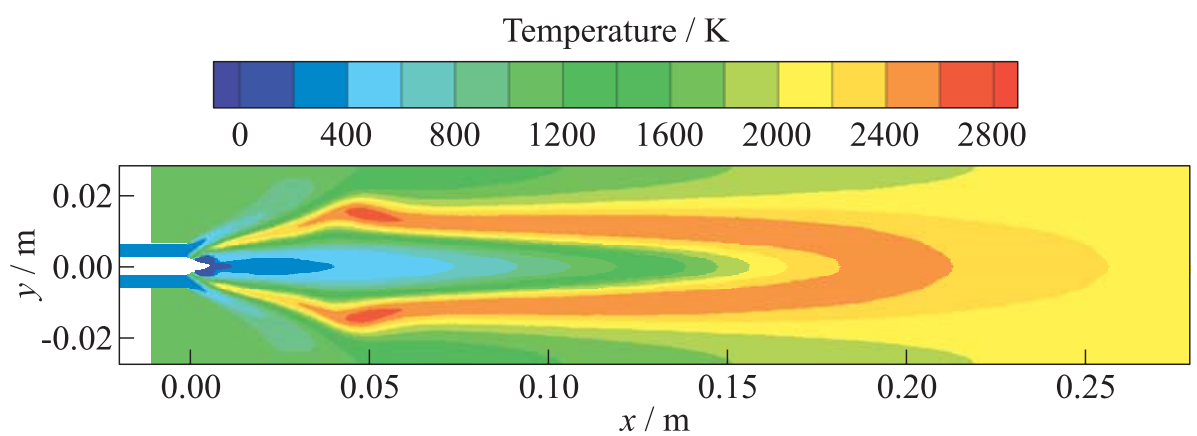

(b)

Figure 4 Mean mixture fraction field $(a)$ and mean temperature field $(b)$ as obtained from the reactive numerical simulation of the Mascotte test case. (Refer Izard and Mura, p. 219.) 
the central spray of LOx droplets [42]. These two specific features were clearly recovered by the present numerical simulations: the cryogenic flame is attached just downstream of the injector lip that separates the $\mathrm{GH}_{2}$ stream from the $\mathrm{LOx}$ core (Fig. 4). The stream of gaseous hydrogen - that enters the combustion

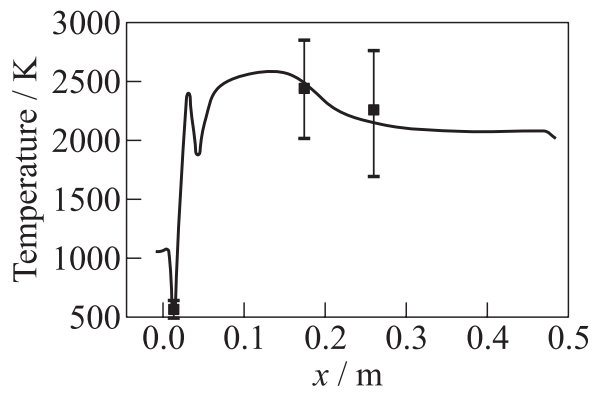

(a)

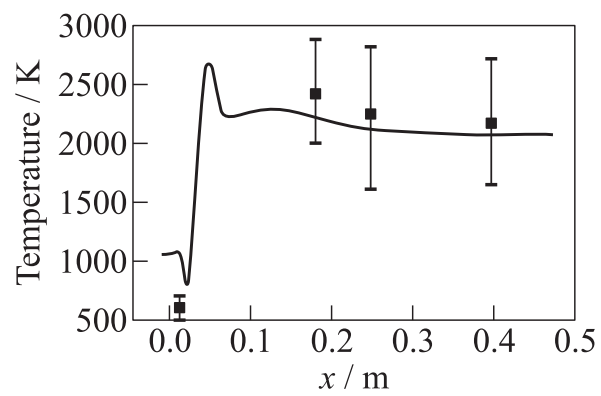

(b)

Figure 5 Axial profiles of mean temperature at $y=10 \mathrm{~mm}(a)$ and $y=15 \mathrm{~mm}(b)$. Comparisons with CARS measurements

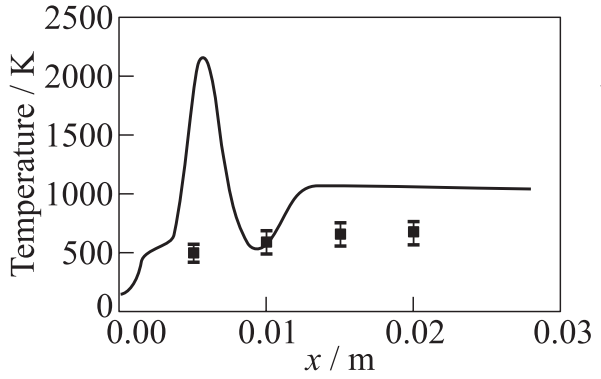

(a)

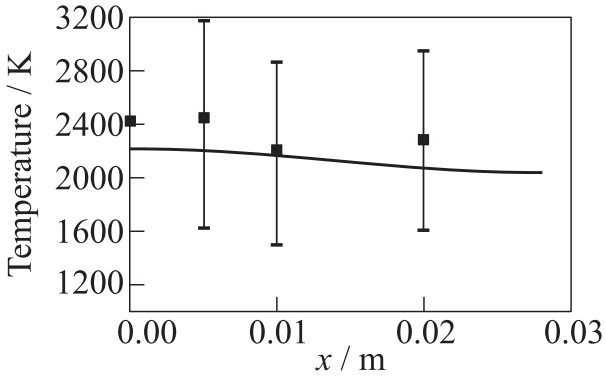

(b)

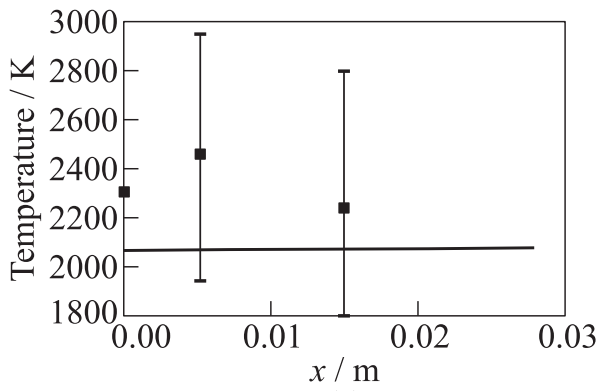

(c)

Figure 6 Radial profiles of mean temperature at $x=10(a), 250(b), 410 \mathrm{~mm}(c)$. Comparisons with CARS measurements 
chamber with a velocity of $320 \mathrm{~m} / \mathrm{s}$ - appears to be strongly deflected towards the walls under the conjugated influence of the droplet stream and the thermal expansion induced by the flame. The flame gives birth to a low-velocity core of cold LOx surrounded by a high-velocity stream of burned gases and unburned hydrogen. Downstream of the position $x=250 \mathrm{~mm}$, all available oxygen has been burned which is consistent with the operating conditions retained for the present test case.

From a more quantitative point of view, axial profiles of mean temperature at $y=10$ and $15 \mathrm{~mm}$ are compared to experimental data in Fig. 5. The standard deviation of the results associated with the experimental measurements are also displayed in this figure. In comparison with available numerical results previously obtained on the same test case, the temperature levels are in satisfactory agreement with experiments. Nevertheless, it seems that the flame length is slightly underestimated by the present computations (Fig. 6). This discrepancy is considered to be more closely related to an overprediction of penetration length of the LOx droplets along the symmetry axis rather than to the closure retained to describe the turbulent flame.

\section{CONCLUDING REMARKS}

The focus of the present study was placed on the description of combustion downstream of a Liquid Oxygen-Gaseous Hydrogen coaxial cryogenic injector. A model of nonpremixed turbulent combustion was used in conjunction with a stochastic Lagrangian simulation of liquid droplets relying on classical atomization models and assuming spherical droplets to simulate spray combustion. Additional terms that take into account the unresolved influence of the dynamics of the vaporizing spray onto the fuel-oxygen composition statistics were also considered and encouraging results were obtained for a reference test case corresponding to the combustion of liquid oxygen with gaseous hydrogen in subcritical cryogenic conditions. The forthcoming steps of the present work will be devoted to:

- the influence of the models retained to represent vaporization and atomization processes; and

- the quantification of the effects of unresolved vaporization-induced fluctuations.

Finally, it is also planned to simulate the rocket engine ignition with special emphasis on the interaction of the central underexpanded torch igniter with peripheral $\mathrm{LOx} / \mathrm{GH}_{2}$ injectors. 


\section{ACKNOWLEDGMENTS}

Financial support from CNES and SNECMA (SAFRAN Group, Space Engines Division) and from CNRS is gratefully acknowledged. The authors are indebted to N. Meyers (SNECMA), D. Saucereau (SNECMA), and B. Vieille (CNES) for fruitful interactions. They also would like to thank the reviewers of their manuscript for valuable suggestions and remarks.

\section{REFERENCES}

1. Williams, F. A. 1958. Spray combustion and atomization. Phys. Fluids 1(6):541-45.

2. Domelevo, K., and L. Sainsaulieu. 1997. A numerical method for the computation of the dispersion of particles by a turbulent gas flow field. J. Comput. Phys. 133:25678.

3. Borghi, R. 1988. Turbulent combustion modelling. Progr. Energy Combust. Sci. $14: 245-92$.

4. Demoulin F.X., and R. Borghi. 2002. Modelling of turbulent spray combustion with application to Diesel like experiment. Combust. Flame 129:281-93.

5. Masquelet, M., S. Menon, Y. Jin, and R. Friedrich. 2009. Simulation of unsteady combustion in a $\mathrm{LOx}-\mathrm{GH}_{2}$ fueled rocket engine. Aerospace Sci. Technol. 13:466-74.

6. Oefelein, J. C. 2005. Thermophysical characteristics of $\mathrm{LOx}-\mathrm{H}_{2}$ flames at supercritical pressure. Proc. Combust. Inst. 30:2929-37.

7. Jay, S., F. Lacas, and S. M. Candel 2006. Combined surface density concepts for dense spray combustion. Combust. Flame 144(3):558-77.

8. Demoulin, F.X., S. Zurbach, and A. Mura. 2009. High pressure supercritical turbulent cryogenic injection and combustion: A single-phase flow modelling proposal. J. Propul. Power 25(2):452-65.

9. Bodèle, E., I. Gökalp, S. Zurbach, and D. Saucereau. 2001. Modeling of Mascotte 10 bars case with THESEE code. 2nd Workshop (International) on Rocket Combustion Modeling Proceedings. Lampoldshausen, Germany.

10. Pourouchottamane, M., F. Dupoirieux, L. Vingert, M. Habiballah, and V. Burnley. 2001. Numerical analysis of the 10 bar Mascotte flow field. 2nd Workshop (International) on Rocket Combustion Modeling Proceedings. Lampoldshausen, Germany.

11. Haidn, O., and M. Habiballah. 2003. Research on high pressure cryogenic combustion. Aerospace Sci. Technol. 7:473-91.

12. Meyers, N., F. Dupoirieux, and M. Habiballah. 2005. An Eulerian model for $\mathrm{LOx} / \mathrm{GH}_{2}$ shear-coaxial jet atomization and combustion. 1st European Conference Aerospace Sciences (EUCASS) Proceedings. Moscow, Russia.

13. Cheng, G. C., and R. C. Farmer. 2006. Validation of a practical dense-spray combustion model for liquid rocket engine injector analyses. J. Propul. Power 22(6):137381. 
14. Izard, J.F., and A. Mura. 2008. A Lagrangian model for non-premixed combustion in supersonic turbulent flows. 18th Shock Interaction Symposium (International) Proceedings. Rouen, France. 207-12.

15. Izard, J.F, G. Lehnasch, and A. Mura. 2009. A Lagrangian model of combustion in high speed flows: Application to scramjet conditions. Combust. Sci. Technol. 181(11):1372-96.

16. Martin, R. 2001. N3SNatur. Version 1.4. Manuel théorique. Simulog Cannes France.

17. Chargy, D. 2008. N3SNATUR-CEDILLE. Version 1.3. Manuel théorique et utilisateur. Cannes, France: INCKA.

18. Vingert, L., M. Habiballah, V. Vuillermoz, and S. Zurbach. 2000. MASCOTTE: A test facility for cryogenic combustion research at high pressure. 51st Astronautical Congress (International) Proceedings. Rio de Janeiro, Brazil.

19. Drew, D.A. 1983. Mathematical modeling of two-phase flows. Ann. Rev. Fluid Mech. 15:261-91.

20. Sirignano, W. A. 1983. Fuel droplet vaporization and spray combustion theory. Progr. Energy Combust. Sci. 9:291-32.

21. Faeth, G.M. 1987. Mixing, transport and combustion in sprays. Progr. Energy Combust. Sci. 13:293-345.

22. Gouesbet, G., and A. Berlemont. 1999. Eulerian and Lagrangian approaches for predicting the behavior of discrete particles in turbulent flows. Progr. Energy Combust. Sci. 25:133-59.

23. Williams, F. A. 1985. Combustion theory. 2nd ed. Benjamin/Cummins Publ. Co.

24. Abramzon, B., and W. A. Sirignano. 1989. Droplet vaporization model for spray combustion calculations. Int. J. Heat Mass Transfer 32(9):1605-18.

25. Dukowicz, J.K. 1980. A particle-fluid numerical model for liquid sprays. J. Comput. Phys. 35:229-53.

26. O'Rourke, P.J. 1989. Statistical properties and numerical implementation of a model for droplets dispersion in a turbulent gas. J. Comput. Phys. 83:345-60.

27. Borghi, R., and M. Gonzalez. 1986. Applications of Lagrangian models to turbulent combustion. Combust. Flame 63:239-50.

28. Obounou, M., M. Gonzalez, and R. Borghi. 1994. A Lagrangian model for predicting turbulent diffusion flames with chemical kinetic effects. Proc. Combust. Inst. 25:1107-13.

29. Villermaux, J., and J. C. Devillon. 1972. Représentation de la coalescence et de la redispersion des domaines de ségrégation dans un fluide par un modèle d'interaction phénoménologique. 2nd Symposium (International) on Chemical Reaction Engineering Proceedings. Amsterdam: Elsevier. B:1-13.

30. Dopazo, C., and E. E. O'Brien. 1974. An approach to the autoignition of a turbulent mixture. Acta Astronautica 1:1239-66.

31. Mura, A., and F. X. Demoulin. 2007. Lagrangian intermittent modeling of turbulent lifted flames. Combust. Theory Modell. 11(2):129-40.

32. Launder, B. E. 1976. Heat and mass transport. In: Turbulence, topics in applied physics. Ed. P. Bradshaw. Berlin, Heidelberg: Springer Verlag. 231-87. 
33. Mura, A., F.X. Demoulin, and R. Borghi. 2004. Turbulent reaction rate in twophase flow flames. European Congress on Computational Methods in Applied Sciences and Engineering ECCOMAS 2004 Proceedings. Jyväskylä, Finland.

34. Borghi, R. 1995. The links between turbulent combustion and spray combustion and their modelling. In: Transport phenomena in combustion. Ed. S. H. Chan. Taylor and Francis. 1:1-18.

35. Guerre, S., R. Bazile, and D. Stepowski. 1996. Conditioned dissipation and average consumption maps in a turbulent non-premixed flame using Planar Laser Induced Fluorescence. Proc. Combust. Inst. 26:105-11.

36. Snyder, R., G. Herding, J. C. Rolon, and S. Candel. 1997. Analysis of flame patterns in cryogenic propellant combustion. Combust. Sci. Technol. 124:331-70.

37. Candel, S., G. Herding, R. Snyder, P. Scouflaire, J. C. Rolon, L. Vingert, M. Habiballah, F. Grisch, M. Péalat, P. Bouchardy, D. Stepowski, A. Cessou, and P. Colin. 1998. Experimental investigation of shear-coaxial cryogenic jet-flame. J. Propul. Power 14:826-34.

38. Demoulin, F.X., P.A. Beau, G. Blokkeel, A. Mura, and R. Borghi. 2007. A new model for turbulent flows with large density fluctuations: Application to liquid atomization. Atomization Sprays 17(4):315-45.

39. Caré, I., and M. Ledoux. 1991. Study of an airblast co-axial atomizer: Experiments and modelling. ICLASS'91 Proceedings. Rouen, France.

40. Gicquel, P., E. Porcheron, and E. Brisson. 1998. Caractérisation expérimentale en combustion d'un brouillard $\mathrm{LOx}-\mathrm{GH}_{2}$ issu d'un injecteur coaxial. Rapport Technique ONERA RT98/6128 DEFA/Y/DMTE.

41. Gicquel, P., and B. Tanguy. 1999. Faisabilité de mesures de vitesses en phase gazeuse dans le foyer Mascotte. Rapport Technique ONERA RT120/6128 DEFA/ Y/DMTE.

42. Juniper, M., A. Tripathi, P. Scouflaire, J. C. Rolon, and S. M. Candel. 2000. The structure of cryogenic flames at elevated pressure. Proc. Combust. Inst. 28:1103-09. 